\title{
Numerical Investigation of Liquid-Liquid Mixing in Modified T Mixer with 3D Obstacles
}

\author{
Md. Readul Mahmud \\ Department of Physical Sciences, Independent University, Bangladesh (IUB), Dhaka-1229, Bangladesh
}

Received: April 15, 2021, Revised: May 08, 2021, Accepted: May 11, 2021, Available Online: June 14, 2021

\begin{abstract}
The fluids inside passive micromixers are laminar in nature and mixing depends primarily on diffusion. Hence mixing efficiency is generally low, and requires a long channel length and longtime compare to active mixers. Various designs of complex channel structures with/without obstacles and three-dimensional geometries have been investigated in the past to obtain an efficient mixing in passive mixers. This work presents a design of a modified $\mathrm{T}$ mixer. To enhance the mixing performance, circular and hexagonal obstacles are introduced inside the modified T mixer. Numerical investigation on mixing and flow characteristics in microchannels is carried out using the computational fluid dynamics (CFD) software ANSYS 15. Mixing in the channels has been analyzed by using Navier-Stokes equations with water-water for a wide range of the Reynolds numbers from 1 to 500 . The results show that the modified $\mathrm{T}$ mixer with circular obstacles has far better mixing performance than the modified $\mathrm{T}$ mixer without obstacles. The reason is that fluids' path length becomes longer due to the presence of obstacles which gives fluids more time to diffuse. For all cases, the modified $\mathrm{T}$ mixer with circular obstacle yields the best mixing efficiency (more than 60\%) at all examined Reynolds numbers. It is also clear that efficiency increase with axial length. Efficiency can be simply improved by adding extra mixing units to provide adequate mixing. The value of the pressure drop is the lowest for the modified $\mathrm{T}$ mixer because there is no obstacle inside the channel. Modified T mixer and modified T mixer with circular obstacle have the lowest and highest mixing cost, respectively. Therefore, the current design of modified $\mathrm{T}$ with circular obstacles can act as an effective and simple passive mixing device for various micromixing applications.
\end{abstract}

Keywords: Micro device, CFD, SAR, Mixing efficiency, Mixing cost.

This work is licensed under a Creative Commons Attribution-Non Commercial 4.0 International License.

\section{Introduction}

Mixing different ingredients is a common scenario of everyday life but achieve good or homogeneous mixing is not always easy. Micro-devices and micro-mixers serve the purpose to achieve excellent mixing in micro-scale [1]. The application of micro-devices is increasing every day in various chemical processes and synthesis [2], particle synthesis, [3] biomedical and biochemical analysis [4], dynamic cell separation [5], Bio Micro-Electro-Mechanical Systems (BioMEMS), and micrototal analysis systems [6]. Micromixers possess a high surfaceto-volume ratio due to their small linear dimensions, a characteristic from which derives most of their advantages over conventional-size chemical processes equipment [7]. In the micro-scale, the flow is mostly laminar at a low Reynolds number and the mixing entirely depends on the molecular diffusion [8]. Hence long length and a long time are required to yield good mixing [9]. Microdevices offer many advantages over traditional equipment such as process safety, low cost to manufacture, better process control, simpler process optimization, rapid design implementation, and easier scale-up through numbering up [7], [10]-[12]. Another major advantage of micromixers is the consumption of significantly less amount of sample and reagent than other known experimental platforms due to its small dimension [13]-[14].

Micromixers are generally divided into two categories, active and passive [15]-[16]. Passive mixers have no active components but use the long channel length and specific geometric configuration to increase the interfacial area between fluids for higher mixing [12]. On contrary, active mixers use active elements to achieve mixing such as acoustic/ultrasonic, dielectrophoretic, electrokinetic time-pulse, pressure perturbation, electro-hydrodynamic, magnetic or thermal techniques to enhance the mixing performance [17]. Though active mixer is more compact and offers higher efficiency [18], passive mixers are economical, convenient, and can easily be incorporated into LOC systems, and protect sensitive bioregents [19].

The earliest and the simplest passive mixers are $\mathrm{T}$ shape and $\mathrm{Y}$ shape micromixers. The detailed evaluation and working principle of passive mixer including T-shaped micromixers have been investigated extensively in recent years [19]-[24]. Many authors investigate the flow regime, the influence of secondary flow, vortex flow, and mixing performance of $\mathrm{T}$ mixers numerically or/and experimentally [2], [11], [25]-[27]. Simple T mixer has low efficiency due to its laminar flow and absence of chaotic advection at low Reynolds number. As a result, good mixing requires long channel length and longtime [28]. To negate this disadvantage, obstacles or grooves are introduced inside the mixers to introduce chaotic advection. Many T mixers have been investigated [12], [29]-[33] which provide an improved mixing quality but incurred a considerably large pressure drop due to the presence of obstacles. Therefore, it is still a challenge to design and optimize $\mathrm{T}$-shaped micromixers with obstacles to achieve higher mixing efficiency with lower mixing costs.

In this present work, a simple $\mathrm{T}$ mixer and a modified $\mathrm{T}$ mixer are presented. The purpose of the work is to improve the efficiency of the modified T mixer by introducing 3D obstacles. Circular-shaped and hexagonal-shaped obstacles are introduced inside the modified $\mathrm{T}$ mixers to enhance the mixing performance. 
Numerical simulation is performed using ANSYS Fluent 15 over a wide range of Reynolds numbers from 1 to 500. The numerical data for a simple T mixer is compared with published work to validate the numerical work. Finally, the overall comparative analysis is performed to propose the best-performing mixer.

\section{Design of Device}

A simple $\mathrm{T}$ shape mixer generally has two inlets and one outlet. The geometry of a simple T mixer investigated by Gianni Orsi et al. [34] is presented in Fig. 1. The inlet channels present a square cross-section, i.e., $W_{i}=H=0.1 \mathrm{~mm}$, whereas the outflow channel has an aspect ratio $2: 1$, i.e., $W_{o}=2 H=$
$0.2 \mathrm{~mm}$. A modified $\mathrm{T}$ shape mixer was designed with same inlets and outlet configuration (Fig. 1). The mixing length is kept constant for both mixers, $L=80 \mathrm{H}=8 \mathrm{~mm}$.

To improve the mixing performance, two different types of 3D obstacles namely, circular and hexagonal shapes are placed inside the modified $\mathrm{T}$ mixer. The proposed modified $\mathrm{T}$ mixer consists of 8 identical elements, each element is $1 \mathrm{~mm}$ long; the minimum and maximum width is $0.1 \mathrm{~mm}$ and $0.2 \mathrm{~mm}$, respectively. Fig. 2 shows the dimension and location of different obstacles inside of an element, as an example of the proposed modified $\mathrm{T}$ mixer.

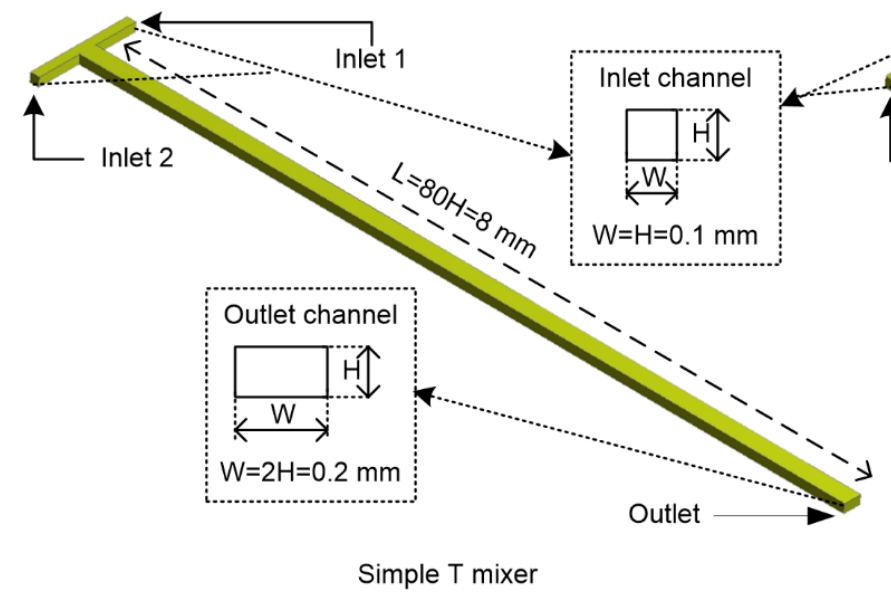

(a)

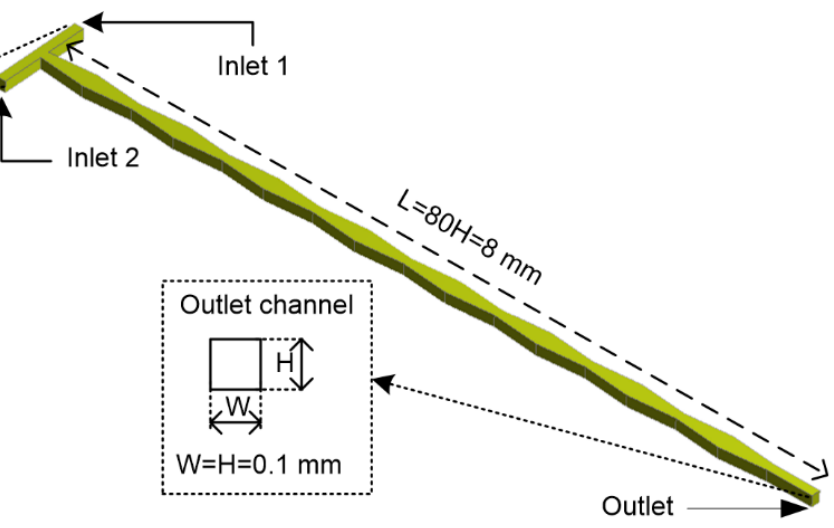

Modified T mixer

(b)

Fig. 1 (a) Simple T mixer and (b) modified T mixer with dimension
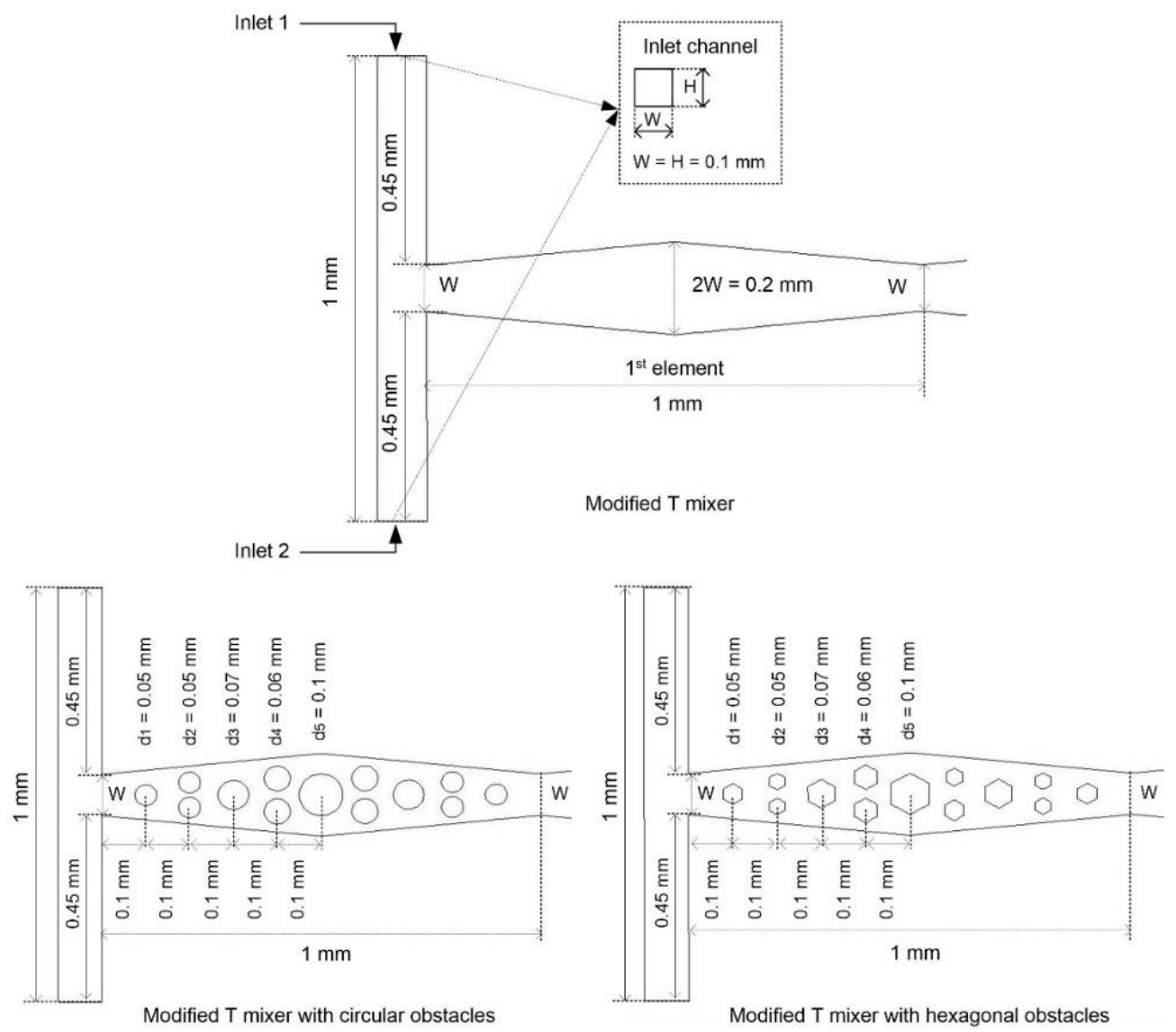

Fig. 2 A single element of modified T, modified $\mathrm{T}$ with circular obstacle and modified $\mathrm{T}$ with hexagonal mixer 


\section{Simulation Setup and Numerical Methodology}

A commercial software ANSYS Fluent 15 is used for numerical simulation purpose. The fluids are considered as Newtonian, steady, incompressible, and the flow field solves using continuity, Navier-Stokes, and advection-diffusion equations as given below [12], [35]-[36]:

$$
\begin{aligned}
& \nabla \cdot V=0 \\
& \rho V \nabla \cdot V=-\nabla P+\mu \nabla^{2} V \\
& J V \cdot \nabla C=D \nabla^{2} C
\end{aligned}
$$

Where $V, \rho, P, \mu, C$, and $D$ are the fluid velocity, fluid density, fluid pressure, fluid viscosity, fluid molar concentration, and diffusivity, respectively.

Numerical simulation is performed considering no-slip velocity condition at all walls, uniform concentration, and two inlets are set to velocity inlet and one outlet is set to pressure outlet with zero (0) gauge pressure [37]. The two inlet fluids are considered to have the same properties as water at $20^{\circ} \mathrm{C}$ with density $\rho=998.2 \mathrm{Kg} / \mathrm{m}^{3}$, dynamics viscosity $\mu=0.001 \mathrm{Pas}$ and diffusivity $D=1 \times 10^{-9} \mathrm{~m}^{2} / \mathrm{s}$ [1], [4]. The fluid concentration at the two inlets is set to 1 and 0 , respectively. The SIMPLEC algorithm is used to couple the velocity and pressure fields [10]. Numerical simulation always affects by artificial diffusive flux and it is impossible to rid of the numerical error completely. But these errors can be reduced by applying a higher-order solving scheme and decreasing mesh size. Hence, second-order upwind scheme have been used for solving the governing equations [38]. The convergence criterion was set $1 \times$ $10^{-6}$ for all parameters.

The Reynolds numbers $(R e)$ are calculated using the following equation using hydraulic diameter $(d)$ [34].

$$
\begin{aligned}
& R e=\frac{\rho V d}{\mu} \\
& d=\frac{2 W H}{W+H}
\end{aligned}
$$

Where $\mathrm{W}$ and $\mathrm{H}$ are the mixing channel width and height, respectively. The mixing index is calculated by using the following equations [39]-[40].

$$
\begin{aligned}
& \sigma=\sqrt{\frac{1}{N} \sum_{i=1}^{N}\left(C_{i}-C_{a v}\right)^{2}} \\
& \eta=1-\sqrt{\frac{\sigma^{2}}{\sigma^{2} \text { max }}}
\end{aligned}
$$

Where, $C_{i}, C_{a v}, \sigma$ and $\sigma_{\max }$ are the concentration at the $\mathrm{i}^{\text {th }}$ node, average concentration, standard deviation, and maximum standard deviation which is 0.5 , respectively. The mixing efficiency is denoted by $\eta$ which ranged from 0 to 1 . Complete mixing is equivalent to 1 and non-mixing is equivalent to 0 .

To have a comprehensive comparative performance of the mixers, mixing cost $\left(M_{C}\right)$ which is the ratio of efficiency to pressure-drop is computed by following equation [19], [41]:

$$
M_{C}=\frac{\eta}{\Delta P}
$$

where $M_{C}, \Delta P$ and $\eta$ denote the mixing cost in $1 / P a$, the pressure drop in $P a$, and the mixing efficiency, respectively.

\subsection{Meshing}

Uniform hexahedral mesh was created by applying the sweep method in the Fluent platform. Fig. 3 shows the grid system of the modified $\mathrm{T}$ mixer and modified $\mathrm{T}$ mixer with circular obstacles, as an example.

The grid independence study was performed for various nodes for all mixers. Fig. 4 shows the grid dependency test of modified $\mathrm{T}$ mixer with circular obstacle for six different number of nodes from $3.45 \times 10^{5}$ to $9.31 \times 10^{5}$. The mixing index was calculated at the outlet at $R e=50$. It is clear that mixing index decreases considerably with the increase of members of nodes, as expected. However, the variation is very small, about $0.98 \%$ in mixing index between $7.11 \times 10^{5}$ to $9.31 \times 10^{5}$ nodes. Hence $7.11 \times 10^{5}$ nodes are used for further analysis of modified $\mathrm{T}$ mixer which will cost-effectively provide acceptable numerical data. Similarly, analyses were performed, and $4.9 \times$ $10^{5}, 5.21 \times 10^{5}, 6.61 \times 10^{5}$ grid cells were chosen for simple $\mathrm{T}$ mixer, modified $\mathrm{T}$ mixer, and modified $\mathrm{T}$ mixer with hexagonal obstacles, respectively.

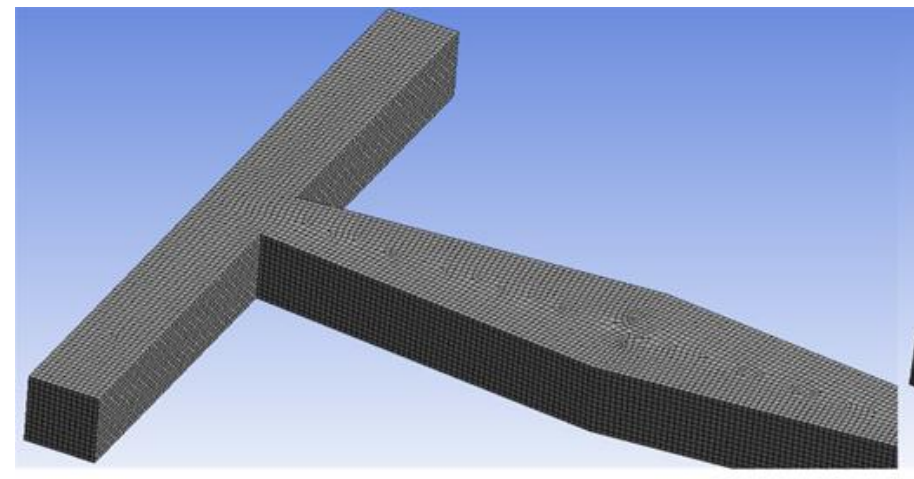

Modified T mixer

(a)

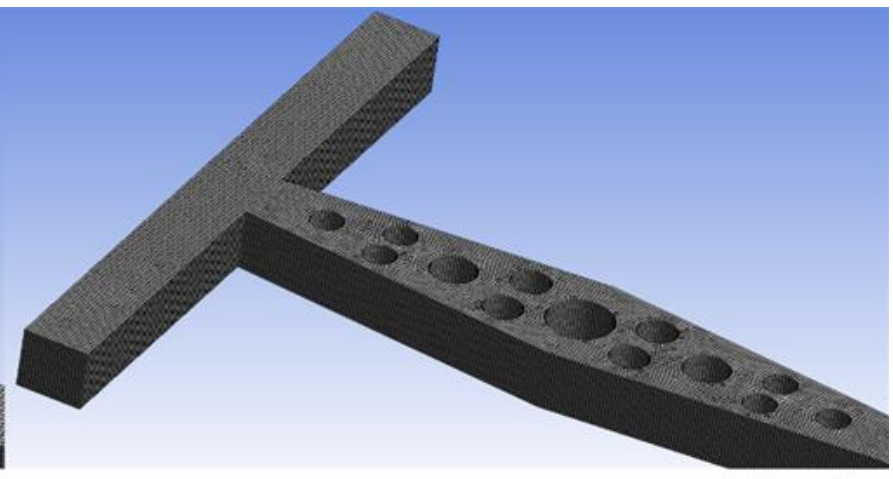

Modified T mixer with circular obstacle

(b)

Fig. 3 Uniform hexagonal grid systems inside the (a) modified $\mathrm{T}$ and (b) modified $\mathrm{T}$ with circular obstacles mixers 


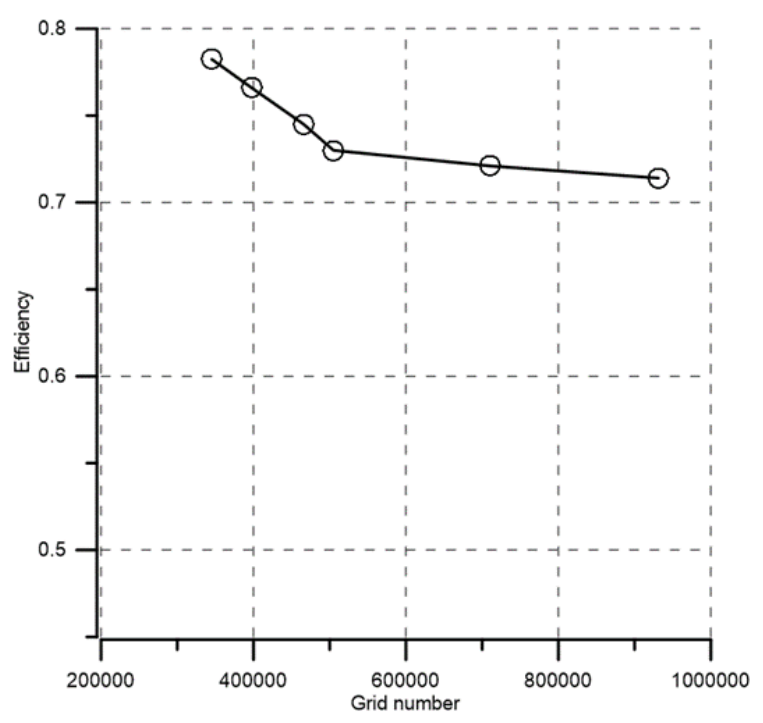

Fig. 4 Dependency of mixing efficiency at the outlet of modified $\mathrm{T}$ mixer with circular obstacles at $R e=50$

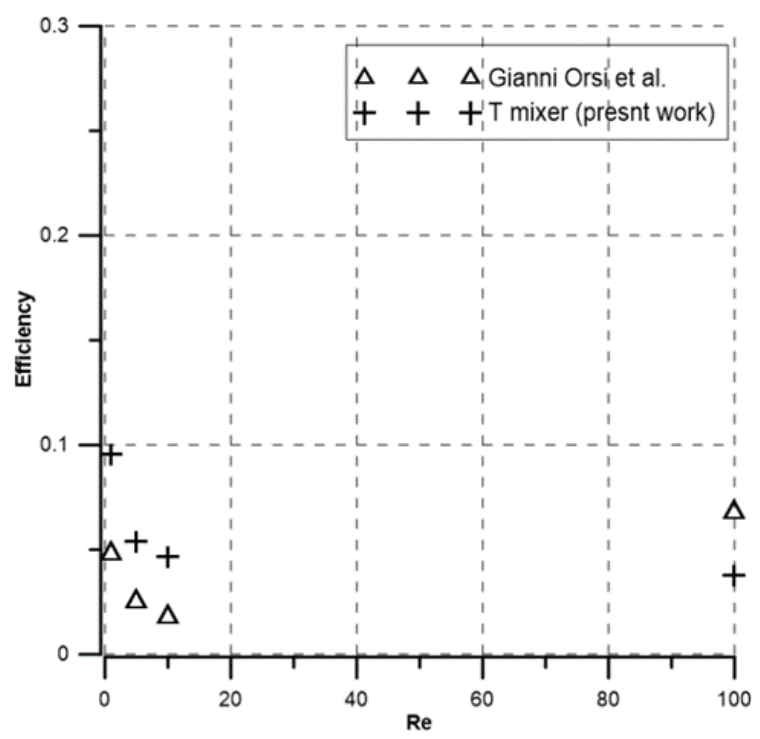

Fig. 5 Comparison of mixing efficiency at a distance of $0.5 \mathrm{~mm}$ $(L=0.5 \mathrm{~mm})$ of a simple T mixer

\section{Results and Discussion}

Since this work involves numerical simulation, qualitative validation of the CFD model was performed by comparing published data. For this purpose, a simple T mixer is constructed keeping the geometrical configuration exactly the same as the published work by Gianni Orsi et al. [36]. The mixing efficiency of a simple T mixer is compared with the published data [36] for water-water mixing and is demonstrated in Fig. 5. The data shows a similar trend and the similarity between them is in an acceptable range because it is quite difficult to replicate exact simulation results due to lack of detailed information. Hence it can be safely assumed that the simulation model is acceptable for the purpose of this work.

Now the comparison between a simple $\mathrm{T}$ mixer and the modified $\mathrm{T}$ mixer is present in Fig. 6. The mixing efficiency is calculated at the outlet for both mixers $(L=8 \mathrm{~mm})$. The efficiency is relatively low for both mixers, less than $30 \%$, but the modified $\mathrm{T}$ mixer shows three times higher efficiency compare to the simple $\mathrm{T}$ mixer. Hence modified $\mathrm{T}$ mixer is chosen for further analysis with different shaped obstacles.

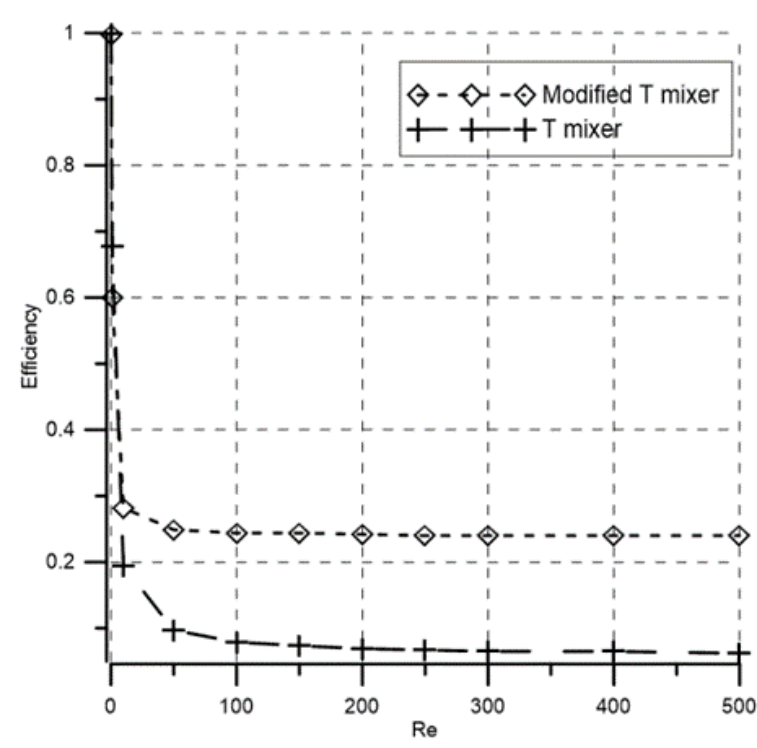

Fig. 6 Mixing efficiency at the outlet $(L=8.5 \mathrm{~mm})$ of a simple $\mathrm{T}$ and the modified $\mathrm{T}$ mixer

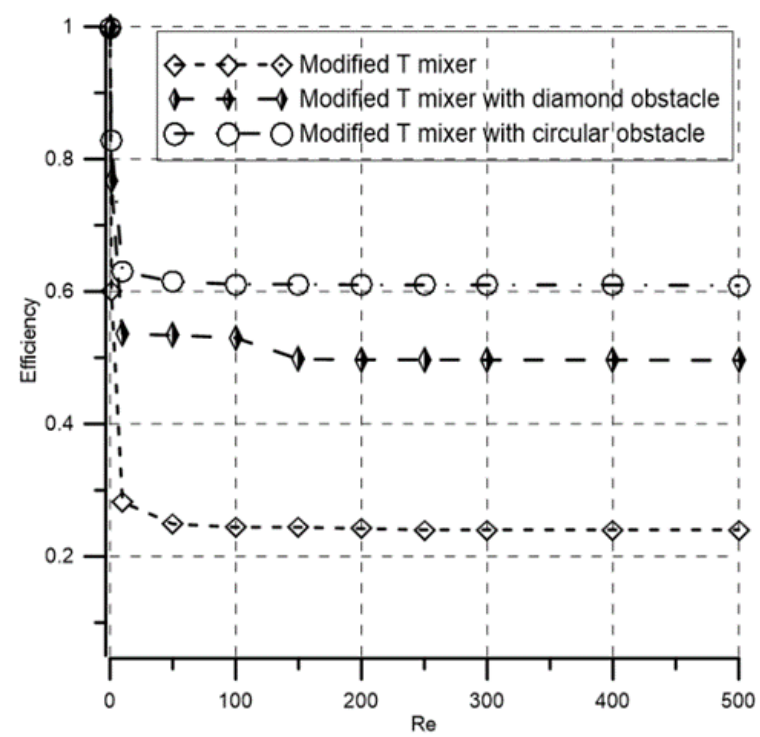

Fig. 7 Mixing efficiency at the outlet of modified T mixers

To improve the mixing performance, circular-shaped and hexagonal-shaped obstacles are places inside the modified $\mathrm{T}$ mixer. Fig. 7 presents the progress of mixing at different Reynolds numbers inside the modified $\mathrm{T}$ mixer with circular obstacles and modified $\mathrm{T}$ mixer with hexagonal obstacles. The efficiency is computed at the outlet of all mixers on the XY plane for $1 \leq R e \leq 500$. All mixers have good mixing efficiency, (more than $70 \%$ ) at low Reynolds numbers $(R e \leq 1)$. It is also found that mixing efficiency of three mixers subsequently decreases with the increase of Reynolds numbers. T mixer with circular obstacle shows the highest efficiency $(\eta>60 \%)$ throughout the Reynolds numbers range compare to the other two mixers. At low Reynolds numbers $(R e \leq 1)$ fluids have more time to mix inside the mixer channel, hence all mixers show good efficiency. At high Reynolds numbers ( $R e \geq 10)$, mixing time decrease and as a consequence efficiency decreases as well. The fluids concentration inside the three mixers at $R e=1$ and $R e=100$ are presented in the Fig. 8. It is clear that fluids homogeneity decreases at higher Reynolds numbers $(R e=$ 100) which indicate efficiency will be higher at low Reynolds number $(R e=1)$ for all three mixers, as confirmed in Fig. 7. 
The distributions of the mass fraction of liquid 1 (water) along the horizontal mid-line, i.e. $x=8 \mathrm{~mm}, y=0, z=0.05 \mathrm{~mm}$ at the outlet of the four micromixers as shown in Fig. 9. A constant value of the mass fraction equal to 0.5 represents ideal mixing. The distributions of the mass fraction of liquid 1 for the modified $\mathrm{T}$ mixer with hexagonal and circular obstacles are close to the ideal distribution, at the minimum and maximum Reynolds number compare to the modified $\mathrm{T}$ mixer.

The lowest mass fraction values correspond to the modified $\mathrm{T}$ mixer with circular obstacle exhibiting the highest mixing efficiency among the investigated micromixers corresponds to Fig. 7. The change in mass fraction of liquid 1 becomes steeper as the Reynolds number increases for all mixers. This implies a lower mixing performance at higher Reynolds numbers.

The fluids streamlines presented in Fig. 10 show flow patterns of the three micromixers at $R e=100$. In case of modified T mixers, the streamlines were parallel and smooth, but streamlines become rough inside the rest two mixers. The two modified $\mathrm{T}$ micromixers with obstructions show the split-andrecombination streamlines, which increase the path length of the fluids and responsible for good mixing compared to the modified T mixer.

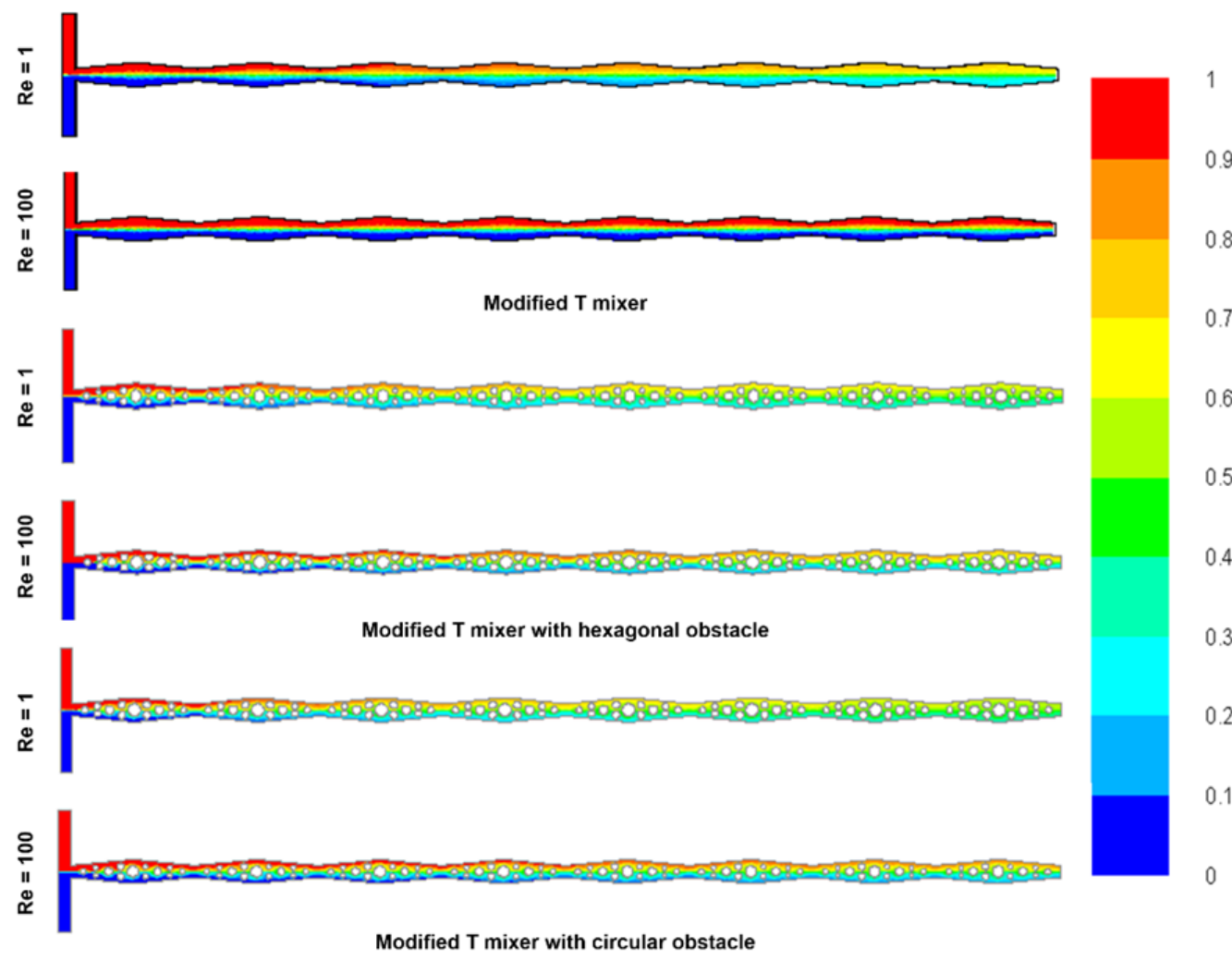

Fig. 8 Concentration of mass fraction of liquids along the channel length of modified $\mathrm{T}$ mixer

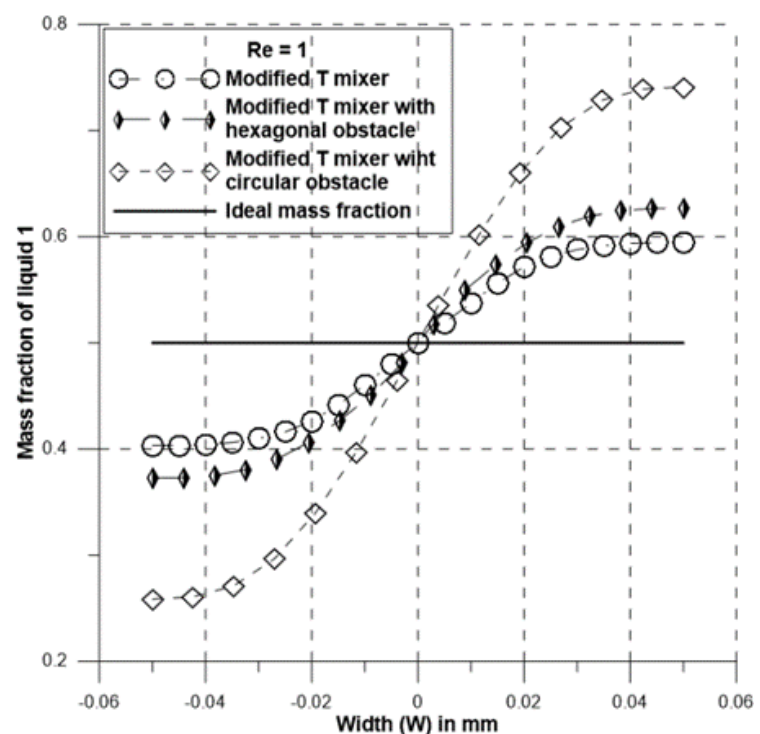

(a)

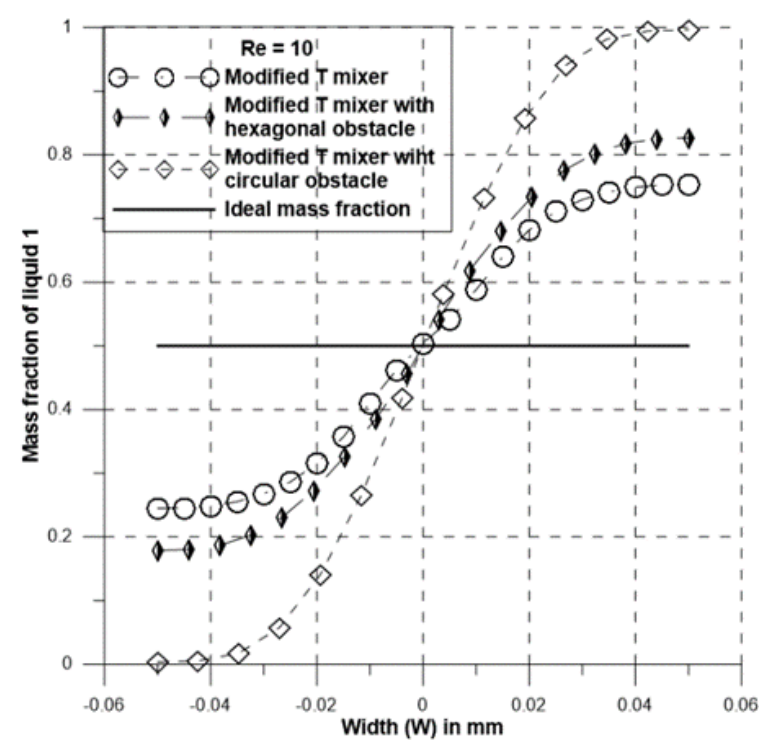

(b)

Fig. 9 Distributaries of mass fraction of liquid 1 along the horizontal mid-line $(\mathrm{z}=0.05 \mathrm{~mm})$ at the outlet of modified $\mathrm{T}$ mixers at (a) $R e=1$ and (b) $R e=10$ 

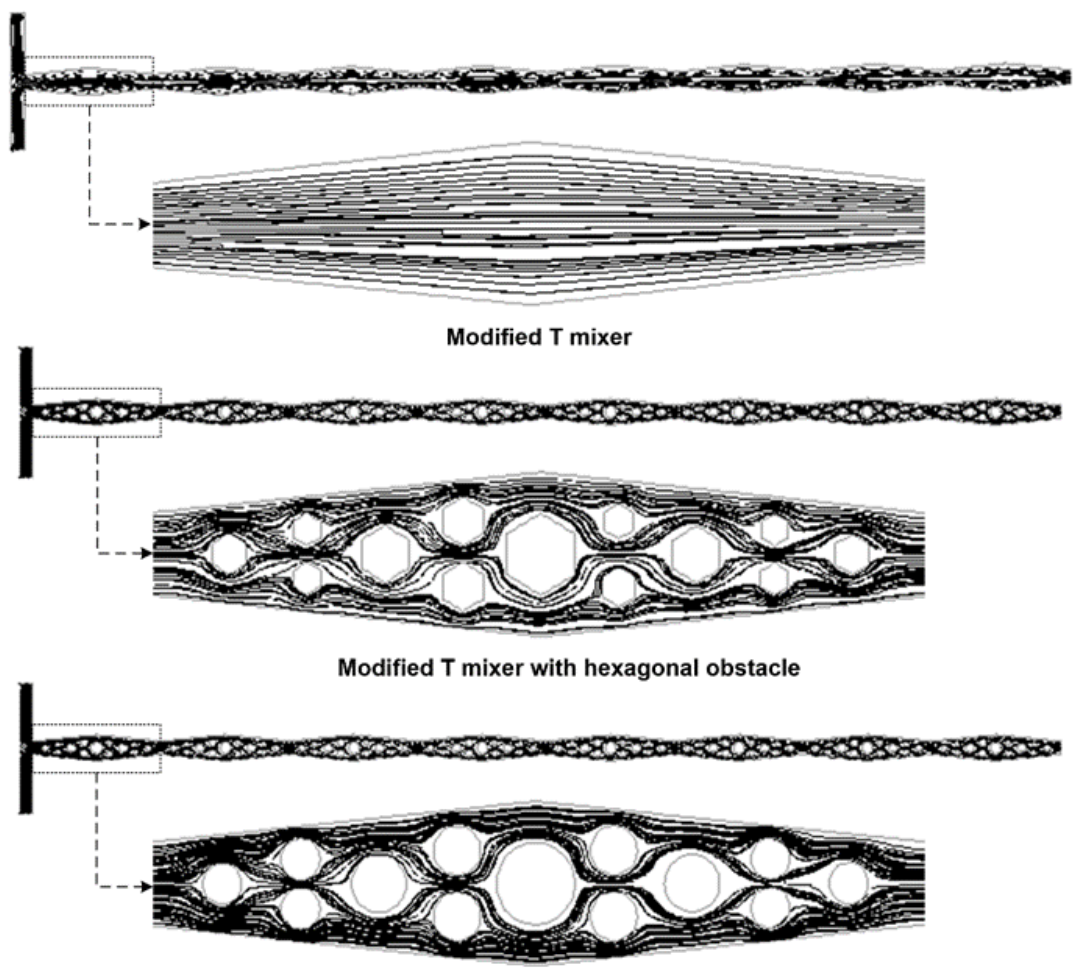

Modified T mixer with circular obstacle

Fig. 10 Fluids streamline inside the modified $\mathrm{T}$ mixers at $R e=100$

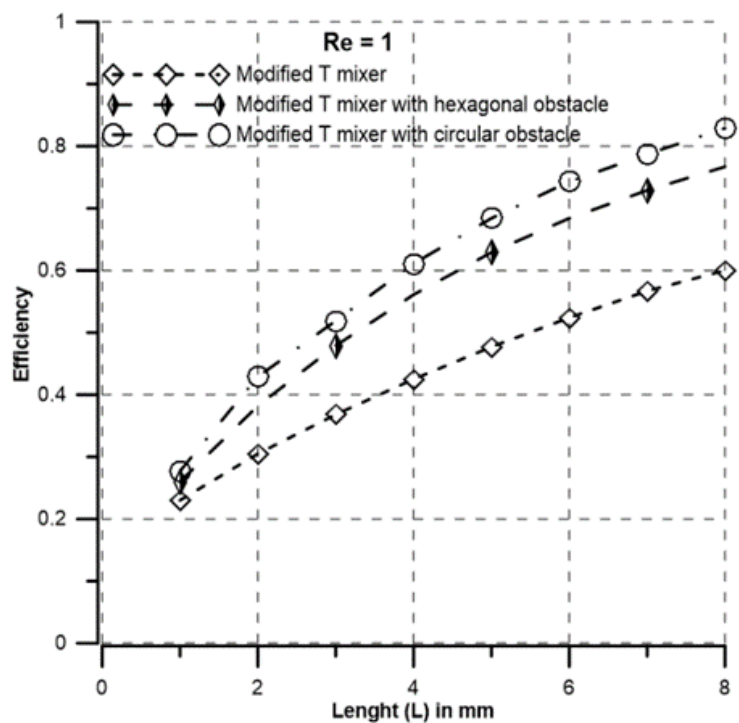

(a)

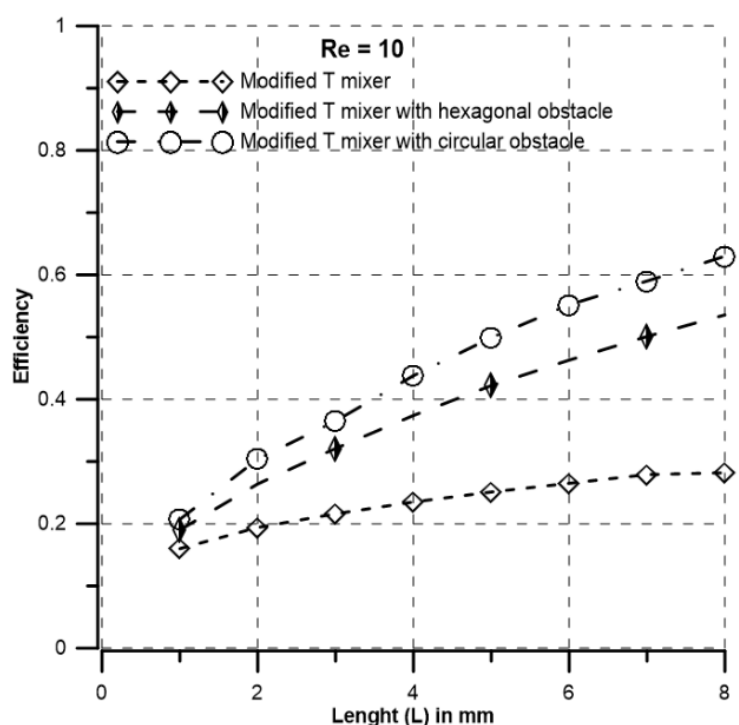

(b)

Fig. 11 Mixing efficiency along the length of modified $\mathrm{T}$ mixers at (a) $R e=1$ and (b) $R e=10$

Mixing efficiency is calculated at several cross-sections along the three micromixer channels at different Reynolds numbers. The efficiency along the axial length at $R e=1$ and $R e=100$ is presented in Fig. 11. The highest mixing indices correspond to the $\mathrm{T}$ mixer with circular obstacles. Modified T mixer shows the lowest efficiency among the three mixers because of the absence of obstacles. Efficiency increases with the increase of channel length due to a longer mixing time for diffusion.

Efficiency also decreases at a higher Reynolds number, which corresponds to Fig. 7. The two new micromixer designs, modified $\mathrm{T}$ and modified $\mathrm{T}$ with hexagonal obstacles have mixing indices of $60 \%$ and $77 \%$ respectively, at the outlet for $R e=1$. Whereas, the efficiency is $82 \%$ in the case of the modified T mixer with circular obstacles. At $R e=10$, efficiency decrease to $28 \%, 53 \%$, and $63 \%$ at the outlet of modified T, modified $\mathrm{T}$ with hexagonal obstacles, and modified $\mathrm{T}$ with circular obstacles, respectively.

The pressure-drop variation as a function of Reynolds numbers for micromixers is shown in Fig. 12. The energy required to drive the fluid from the inlet to the outlet depends on the pressure drop which in turn increases with an increase in Reynolds numbers and vice versa. The lowest pressure drop is observed for the modified $\mathrm{T}$ mixer due to the absence of obstacles. Whereas, modified $\mathrm{T}$ mixer with circular obstacles shows the highest pressure drop. To have better overall mixing performance, a parameter named, mixing cost is computed. The best design of a micromixer can be determined by computing the 
mixing cost for three micromixers. The computed mixing cost for three mixers at $R e=1$ and $R e=100$ is present in Fig. 13 . The mixing cost of the modified $\mathrm{T}$ mixer with circular obstacles is the lowest one compare to the studied three mixers. Modified $\mathrm{T}$ mixer with circular obstacles, has the highest efficiency and lowest mixing cost, indicates the best performing mixers among three micromixers.

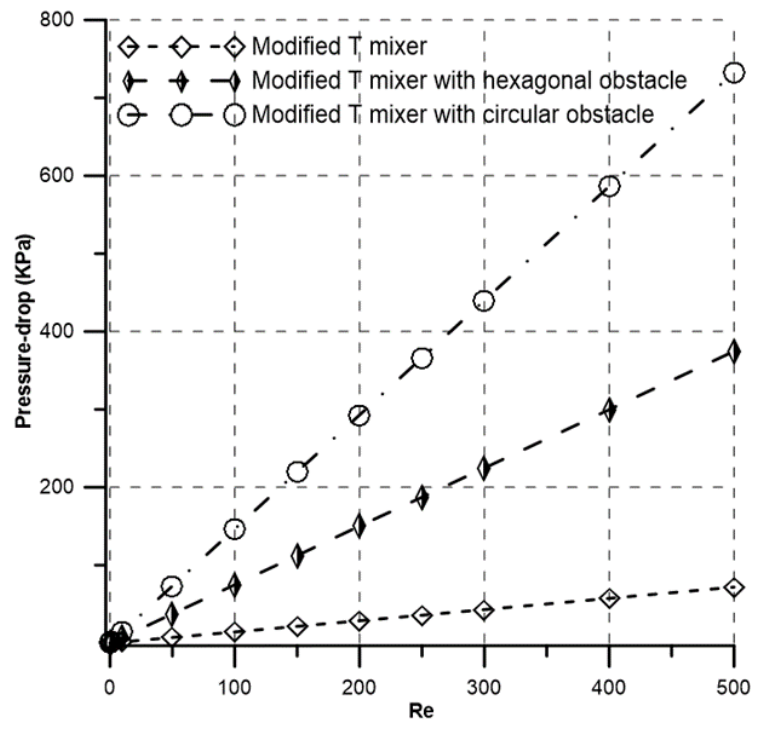

Fig. 12: Pressure-drop at the outlet of modified T mixers
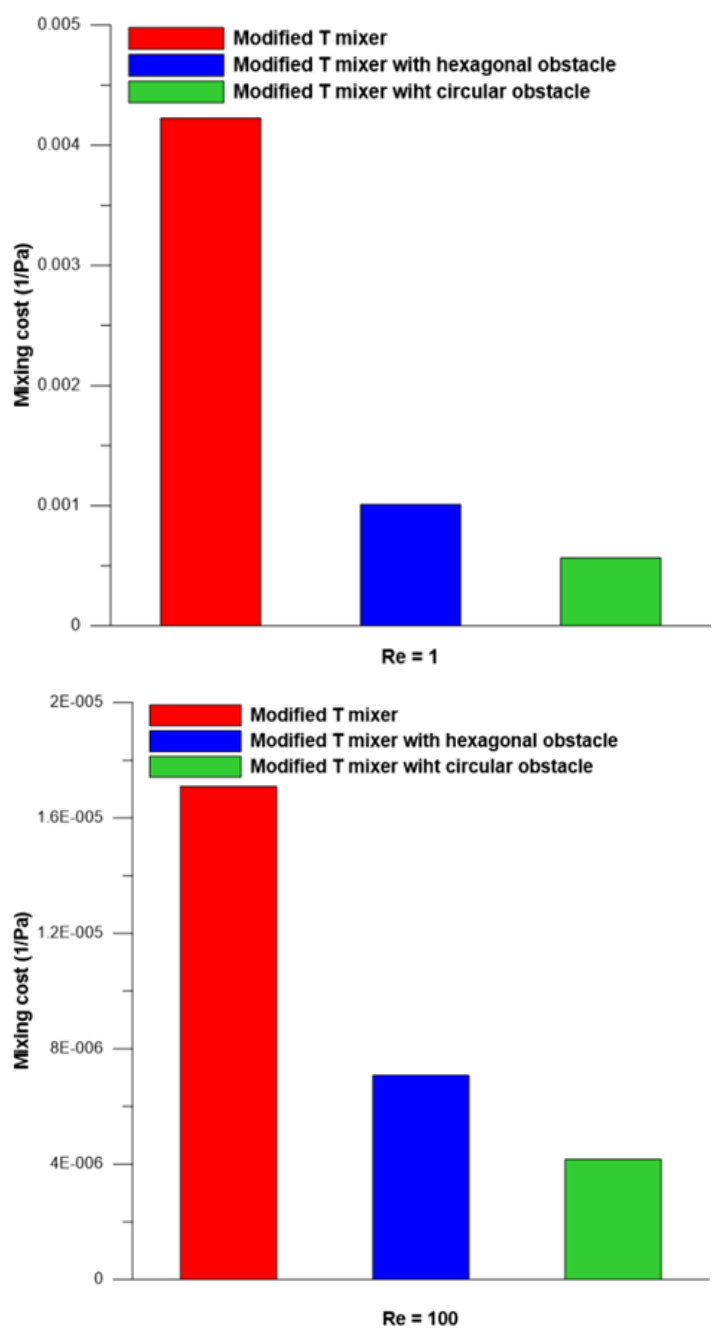

Fig. 13: Mixing cost of modified T mixers at (a) $\operatorname{Re}=1$ and (b) $\mathrm{Re}=100$

\section{Conclusion}

$\mathrm{T}$ mixer is one of the earliest and simplest passive micromixer designs. It is easy to construct and apply in various applications but it has the limitation of poor mixing except at very low Reynolds numbers $(R e<1)$. Many $\mathrm{T}$ mixers incorporate 2D and 3D dimensional baffles or obstacles to overcome these limitations. However, pressure drop always increases due to the presence of obstacles. In the present study, a simple $\mathrm{T}$ and a modified $\mathrm{T}$ mixer are designed and analyzed for a wide range of Reynolds numbers by ANSYS Fluent 15. The numerical data yield from the simple T mixer is compared with published results to justify the present numerical work. A small improvement in mixing performance over the Reynolds numbers ranging from 1 to 500 is obtained by the modified $\mathrm{T}$ mixer over a simple $\mathrm{T}$ mixer. Two kinds of obstacles, namely circular and hexagonal are introduced in the modified T mixers to observe the further increase in mixing performance. The modified $\mathrm{T}$ mixer with circular obstacles and modified $\mathrm{T}$ mixer with hexagonal obstacles have shown considerable improvement in mixing, but both designs have no notable effect to improve the secondary flow. However, the path lengths of fluids increase inside the modified $\mathrm{T}$ mixers with obstacles which in turn give fluids more time to mix, and as a consequence efficiency increases at Reynolds numbers at the mid and high range of Reynolds numbers $(10 \leq R e \leq 500)$. Modified $\mathrm{T}$ mixer with circular obstacles shows $60 \%$ more efficiency compare to a simple $\mathrm{T}$ mixer irrespective of Reynolds numbers. Mixing cost is also computed, which allows for a comparison of the performance of all presented micromixers. Though the modified $\mathrm{T}$ mixer with circular obstacles provides the highest pressure drop, it also has the highest efficiency and lowest mixing cost at all examined Reynolds numbers $(1 \leq R e \leq 500)$.

\section{References}

[1] Rahmannezhad, J. and Mirbozorgi, S.A., 2019. CFD analysis and RSM-based design optimization of novel grooved micromixers with obstructions. International Journal of Heat and Mass Transfer, 140, pp.483-497.

[2] Engler, M., Kockmann, N., Kiefer, T. and Woias, P., 2004. Numerical and experimental investigations on liquid mixing in static micromixers. Chemical Engineering Journal, 101(1-3), pp.315-322.

[3] Bothe, D., Stemich, C. and Warnecke, H.J., 2006. Fluid mixing in a T-shaped micro-mixer. Chemical Engineering Science, 61(9), pp.2950-2958.

[4] Schikarski, T., Trzenschiok, H., Peukert, W. and Avila, M., 2019. Inflow boundary conditions determine T-mixer efficiency. Reaction Chemistry \& Engineering, 4(3), pp.559568.

[5] Wang, X.B., Yang, J., Huang, Y., Vykoukal, J., Becker, F.F. and Gascoyne, P.R., 2000. Cell separation by dielectrophoretic fieldflow-fractionation. Analytical Chemistry, 72(4), pp.832-839.

[6] Hossain, S., Ansari, M.A. and Kim, K.Y., 2009. Evaluation of the mixing performance of three passive micromixers. Chemical Engineering Journal, 150(2-3), pp.492-501.

[7] Adeosun, J.T. and Lawal, A., 2009. Numerical and experimental studies of mixing characteristics in a T-junction microchannel using residence-time distribution. Chemical Engineering Science, 64(10), pp.2422-2432.

[8] Shah, I., Kim, S.W., Kim, K., Doh, Y.H. and Choi, K.H., 2019. Experimental and numerical analysis of Y-shaped split and recombination micro-mixer with different mixing units. Chemical Engineering Journal, 358, pp.691-706. 
[9] Viktorov, V., Mahmud, M.R. and Visconte, C., 2015. Comparative analysis of passive micromixers at a wide range of Reynolds numbers. Micromachines, 6(8), pp.1166-1179.

[10] Mouheb, N.A., Malsch, D., Montillet, A., Solliec, C. and Henkel, T., 2012. Numerical and experimental investigations of mixing in T-shaped and cross-shaped micromixers. Chemical Engineering Science, 68(1), pp.278-289.

[11] Hoffmann, M., Schlüter, M. and Räbiger, N., 2006. Experimental investigation of liquid-liquid mixing in T-shaped micro-mixers using $\mu$-LIF and $\mu$-PIV. Chemical Engineering Science, 61(9), pp.2968-2976.

[12] Dundi, T.M., Raju, V.R.K. and Chandramohan, V.P., 2019. Characterization of mixing in an optimized designed $\mathrm{T}-\mathrm{T}$ mixer with cylindrical elements. Chinese Journal of Chemical Engineering, 27(10), pp.2337-2351.

[13] Wong, S.H., Ward, M.C. and Wharton, C.W., 2004. Micro Tmixer as a rapid mixing micromixer. Sensors and Actuators B: Chemical, 100(3), pp.359-379.

[14] Shamloo, A., Vatankhah, P. and Akbari, A., 2017. Analyzing mixing quality in a curved centrifugal micromixer through numerical simulation. Chemical Engineering and Processing: Process Intensification, 116, pp.9-16.

[15] Mondal, B., Mehta, S.K., Patowari, P.K. and Pati, S., 2019. Numerical study of mixing in wavy micromixers: comparison between raccoon and serpentine mixer. Chemical Engineering and Processing: Process Intensification, 136, pp.44-61.

[16] Gidde, R., 2020. On the study of teardrop shaped split and collision (TS-SAC) micromixers with balanced and unbalanced split of subchannels. International Journal of Modelling and Simulation, pp.1-10.

[17] Huang, J.J., Lo, Y.J., Hsieh, C.M., Lei, U., Li, C.I. and Huang, C.W., 2011, February. An electro-thermal micro mixer. In 2011 6th IEEE International Conference on Nano/Micro Engineered and Molecular Systems (pp. 919-922). IEEE.

[18] Chen, X. and Zhang, L., 2017. A review on micromixers actuated with magnetic nanomaterials. Microchimica Acta, 184(10), pp.3639-3649.

[19] Raza, W., Hossain, S. and Kim, K.Y., 2020. A review of passive micromixers with a comparative analysis. Micromachines, 11(5), p.455.

[20] Cai, G., Xue, L., Zhang, H. and Lin, J., 2017. A review on micromixers. Micromachines, 8(9), p.274.

[21] Nam-Trung, N. and Zhigang, W., 2005. Micromixers-a review. Journal of Micromechanics and Microengineering, 15(2), p.R1.

[22] Lee, C.Y., Wang, W.T., Liu, C.C. and Fu, L.M., 2016. Passive mixers in microfluidic systems: A review. Chemical Engineering Journal, 288, pp.146-160.

[23] Barabash, V.M., Abiev, R.S. and Kulov, N.N., 2018. Theory and practice of mixing: a review. Theoretical Foundations of Chemical Engineering, 52(4), pp.473-487.

[24] Lee, C.Y. and Fu, L.M., 2018. Recent advances and applications of micromixers. Sensors and Actuators B: Chemical, 259, pp.677-702.

[25] Bothe, D., Stemich, C. and Warnecke, H.J., 2008. Computation of scales and quality of mixing in a T-shaped microreactor. Computers \& Chemical Engineering, 32(1-2), pp.108-114.

[26] Bothe, D., Lojewski, A. and Warnecke, H.J., 2011. Fully resolved numerical simulation of reactive mixing in a $\mathrm{T}$-shaped micromixer using parabolized species equations. Chemical Engineering Science, 66(24), pp.6424-6440.

[27] Galletti, C., Roudgar, M., Brunazzi, E. and Mauri, R., 2012. Effect of inlet conditions on the engulfment pattern in a Tshaped micro-mixer. Chemical Engineering Journal, 185, pp.300-313.

[28] Nimafar, M., Viktorov, V. and Martinelli, M., 2012. Experimental comparative mixing performance of passive micromixers with $\mathrm{H}$-shaped sub-channels. Chemical Engineering Science, 76, pp.37-44.

[29] Chen, L., Wang, G., Lim, C., Seong, G.H., Choo, J., Lee, E.K., Kang, S.H. and Song, J.M., 2009. Evaluation of passive mixing behaviors in a pillar obstruction poly (dimethylsiloxane) microfluidic mixer using fluorescence microscopy. Microfluidics and Nanofluidics, 7(2), pp.267-273.

[30] Tseng, L.Y., Yang, A.S., Lee, C.Y. and Hsieh, C.Y., 2011. CFDbased optimization of a diamond-obstacles inserted micromixer with boundary protrusions. Engineering Applications of Computational Fluid Mechanics, 5(2), pp.210-222.

[31] Fang, Y., Ye, Y., Shen, R., Zhu, P., Guo, R., Hu, Y. and Wu, L., 2012. Mixing enhancement by simple periodic geometric features in microchannels. Chemical Engineering Journal, 187, pp.306-310.

[32] Ansari, M.A., Kim, K.Y. and Kim, S.M., 2018. Numerical and experimental study on mixing performances of simple and vortex micro T-mixers. Micromachines, 9(5), p.204.

[33] Santana, H.S., Silva Jr, J.L., Tortola, D.S. and Taranto, O.P., 2018. Transesterification of sunflower oil in microchannels with circular obstructions. Chinese Journal of Chemical Engineering, 26(4), pp.852-863.

[34] Orsi, G., Roudgar, M., Brunazzi, E., Galletti, C. and Mauri, R., 2013. Water-ethanol mixing in T-shaped microdevices. Chemical Engineering Science, 95, pp.174-183.

[35] Jeguirim, M., Belhachemi, M., Limousy, L. and Bennici, S., 2018. Adsorption/reduction of nitrogen dioxide on activated carbons: textural properties versus surface chemistry-a review. Chemical Engineering Journal, 347, pp.493-504.

[36] Cortes-Quiroz, C.A., Azarbadegan, A. and Zangeneh, M., 2017. Effect of channel aspect ratio of 3-D T-mixer on flow patterns and convective mixing for a wide range of Reynolds number. Sensors and Actuators B: Chemical, 239, pp.1153-1176.

[37] Mariotti, A., Galletti, C., Salvetti, M.V. and Brunazzi, E., 2019. Unsteady flow regimes in a T-shaped micromixer: Mixing and characteristic frequencies. Industrial \& Engineering Chemistry Research, 58(29), pp.13340-13356.

[38] Gobby, D., Angeli, P. and Gavriilidis, A., 2001. Mixing characteristics of T-type microfluidic mixers. Journal of Micromechanics and Microengineering, 11(2), p.126.

[39] Viktorov, V., Mahmud, M.R. and Visconte, C., 2016. Design and characterization of a new HC passive micromixer up to Reynolds number 100. Chemical Engineering Research and Design, 108, pp.152-163.

[40] Shinde, A.B., Patil, A.V. and Patil, V.B., 2020. Enhance the mixing performance of water and ethanol at micro level using geometrical modifications. Materials Today: Proceedings.

[41] Chung, C.K. and Shih, T.R., 2007. A rhombic micromixer with asymmetrical flow for enhancing mixing. Journal of Micromechanics and Microengineering, 17(12), p.2495. 